\title{
Experimentelle Untersuchung des Wärmeübergangs beim Sieden binärer Stoffgemische aus Aceton und Ethanol
}

\author{
J. Schmadl und R. Wittl
}

\section{Einleitung}

Das Sieden und besonders das in dieser Arbeit ausschließlich behandelte vollausgebildete Blasensieden ist immer dann die bevorzugte Form der Wärmeübertragung an Flüssigkeiten, wenn hohe Wärmeströme pro Heizflächeneinheit und pro K Heizwandüberhitzung übertragen werden sollen. In vielen Fällen werden dabei nicht reine Stoffe, sondern Stoffgemische eingesetzt, z. B. bei der Kühlung von Chemiereaktoren oder bei der thermischen Trennung von Stoffgemischen (Destillation, Rektifikation und Eindampfung zwecks Kristallisation). Dabei ist zu berücksichtigen, dass der Wärmeübergangskoeffizient beim Blasensieden von Gemischen wegen des kombinierten Wärme- und Stoffübergangs in der Regel kleiner als bei reinen Stoffen ist. Besonders bei hohen Wärmestromdichten, hohen Drücken und hohen relativen Flüchtigkeiten der beteiligten Stoffkomponenten kann dieser „Gemischeffekt“ den Wärmeübergangskoeffizienten um Faktor 10 und mehr herabsetzen, was in der Apparateauslegung berücksichtigt werden Anvisg. $-\bar{x}_{1}+q$ hauptsächlich auf die bevorzugte Verdampfung der leichter siedenden Komponente an der Heizwand zurückgefuihrt: Beim Verdampfen reichert sich die schwerer siedende Komponente in der wandnahen Grenzschicht an und die Siedetemperatur erhöht sich, entsprechend auch die Wandtemperatur. Geht man von Phasengleichgewicht zwischen siedender Flüssigkeit und entstehenden Dampfblasen aus, was bei nicht extrem hohen Wärmestromdichten angenommen werden darf, lassen sich Konzentrationen und Temperaturen des Mikrozustandes an der Heizfläche prinzipiell wie in Abb. 1 darstellen: Durch die Verarmung der Flüssigkeit an leichter Siedendem ist die Konzentration an der Heizfläche $\bar{x}_{1, P h}$ kleiner als die mittlere Fluissigkeitskonzentration $\bar{x}_{1, \text { mix }}$ im Pool. Deshalb ist, dem Verlauf der Siedelinie folgend, die Siedetemperatur an der Phasengrenze $\mathrm{T}_{\mathrm{Ph}}$ höher als die im Fluissigkeitspool $\mathrm{T}_{\mathrm{S}}$. Das führt zu einer Verminderung des Wärmeübergangskoeffizienten, der wie üblich als Verhältnis aus der Wärmestromdichte $\dot{q}$ und der Temperaturdifferenz $\Delta \mathrm{T}$ zwischen Heizwand $\mathrm{T}_{\mathrm{W}}$ und siedender Flüssigkeit $\mathrm{T}_{\mathrm{S}}$ in genügender Entfernung von der Heizwand gebildet wird:

Für Gemische lässt sich die Wandüberhitzung $\Delta T_{\text {mix }}$ in zwei Anteile zerlegen, ein Anteil , der zur Blasenbildung notwendig ist, und ein Exzessanteil in der wegen der Verarmung der Grenzschicht an leichter Siedenden gemäß Abb. 2 zustande kommt:

$$
\Delta T_{\text {mix }}=\underbrace{T_{W}-T_{P h}}_{\Delta T_{\text {id }}}+\underbrace{T_{P h}-T_{S}}_{\Delta T^{E}}=\Delta T_{i d}+\Delta T^{E}>\Delta T_{\text {id }} \cong \Delta T_{\text {reinestoffe }}
$$

In welchem Maße sich der Wärmeübergang verschlechtert, wird also dadurch bestimmt, um wieviel größer die Überhitzung des Gemisches in der Mikroschicht an der Heizfläche gegenüber dem Rest der Flüssigkeit $T_{P h}-T_{S}=\Delta T^{E}$ ist. Der Anteil $\Delta T_{i d}$ kann als gewichteter Mittelwert der analogen Wandüberhitzungen für die reinen Komponenten betrachtet werden:

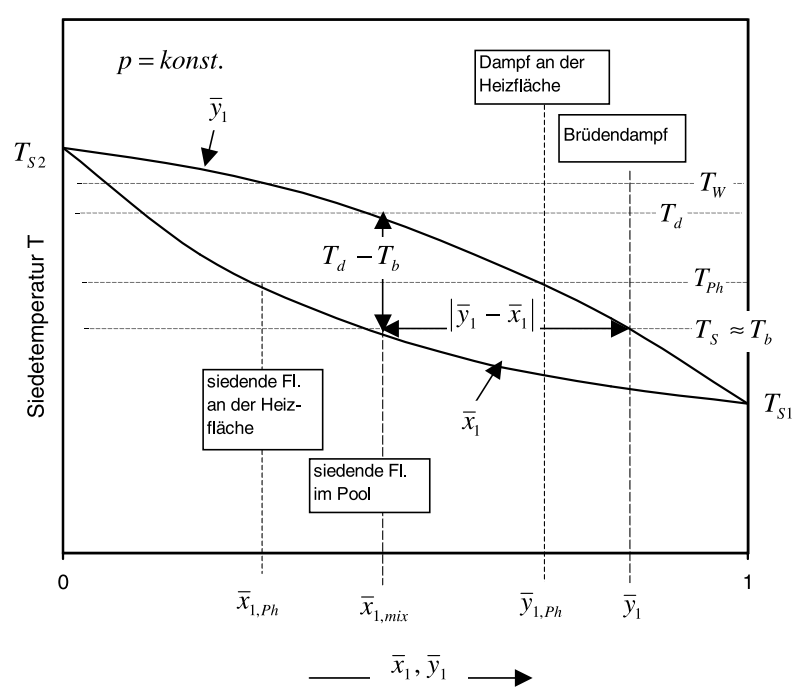

Abb. 1: Qualitative Darstellung der Temperaturen und Konzentrationen bei der Blasenbildung einer siedenden Zweistoffmischung der Konzentration $\bar{x}_{1, \text { mix }}$ an einer beheizten Wand im isobaren Phasengleichgewichtsdia-gramm Dampf/Flüssigkeit.

Stephan und Körner [1] verwenden den molanteilig gewichteten Mittelwert der Wanduiberhitzung der reinen Stoffe $\Delta T_{i d}$ nach Gleichung (3) als Bezugsgröße und korrelieren diese für binäre Gemische mit der Konzentrationsdifferenz zwischen Dampf und siedender Flüssigkeit für die leichtersiedende Komponente 1 nach Abb.1:

Darin ist ist die effektive Heizflächenüberhitzung beim Gemischsieden und $\mathrm{C}$ eine lineare Druckfunktion, die an Messwerte angepasst wird.

Andere Autoren, z. B. Happel [2] bei niedrigen Drücken und Schmadl [3] bei hohen Drücken, korrelieren das Verhältnis aus dem gemessenen -Wert der binären Mi- 
schung und dem aus den Reinstoffdaten molanteilig gemittelten -Wert als Maß für die relative Verschlechterung des Wärmübergangs mit derselben Konzentrationsdifferenz

Darin sind und die entsprechende Reinstoffwerte, nach Happel beim absoluten Druck , nach Schmadl beim normierten Druck $\quad=\mathrm{p} / \mathrm{p}_{\mathrm{k}}$ der Mischung. Entsprechend werden für die Beurteilung des Gemischeffektes auch in dieser Arbeit zwei, den Gleichungen (4) und (5) entsprechende normierte Wärmeübergangskoeffizienten verwendet:

$$
\begin{aligned}
& \frac{\alpha}{\hat{\alpha}}=\frac{\dot{q} / \Delta T_{m i x}}{\dot{q} /\left(x_{1} \cdot \Delta T_{1}+x_{2} \cdot \Delta T_{2}\right)} \\
& \text { und } \frac{\alpha}{\alpha_{i d}}=\frac{\dot{q} / \Delta T_{m i x}}{x_{1} \cdot \alpha_{1}+x_{2} \cdot \alpha_{2}}
\end{aligned}
$$

Ausgangspunkt für die Aufgabenstellung dieser Arbeit war, dass fuir das Stoffpaar Aceton-Ethanol in [4] nur eingeschränkt verwendbare Berechnungsmöglichkeiten für den Wärmeübergangskoeffizienten angegeben sind. Sowohl in [4] wie auch in [5] wird darauf hingewiesen, dass die Gleichung von Stephan und Körner für dieses Stoffpaar nur eingeschränkt anwendbar ist, da für die Korrelation mit den experimentellen Daten von Bonilla und Perry aus dem Jahre 1945 [6] und Alpay aus dem Jahre 1988 [7] nicht ausreichend experimentelle Daten zur Verfuigung gestanden hatten. Ziel dieser Arbeit war deshalb, die aus der Literatur bekannten Messergebnisse fuir Aceton/Ethanol-Mischungen zu ergänzen durch neue Messungen über einen weiteren Bereich des Druckes und der Wärmestromdichte, um die Gleichung von Stephan und Körner überprüfen zu können. Dazu werden in dieser Arbeit die experimentellen Ergebnisse dargestellt, die Korrelationsergebnisse sind in der Folgearbeit [10] enthalten.

\section{Apparatur und Versuchsdurchführung}

Verwendete Apparatur und Messtechnik sind in einer früheren Arbeit [8] ausfuihrlich beschrieben. Sie besteht aus einem Versuchsstoffkreislauf mit Siedekessel, Kondensator sowie Verbindungsleitungen, der sich in einer Klimazelle befindet. Die Messungen wurden an einem elektrisch beheizten, horizontal in der siedenden Fluissigkeit fixierten Versuchsrohr aus Kupfer (Durchmesser $8 \mathrm{~mm}$, beheizte Länge $200 \mathrm{~mm}$ ) durchgefuihrt.

Das Messprogramm umfasste Wärmeübertragungs- und gaschromatographische Konzentrationsmessungen. Die Wärmeübergangsmessungen wurden im Bereich des voll ausgebildeten Blasensiedens für die reinen Stoffe Aceton und Ethanol und für fünf Gemische mit $\bar{x}_{1}=0,1 ; 0,2$;
0,43; 0,67 und 0,9 bei jeweils vier Drüicken $p=1 ; 4 ; 7$ und 10 bar, und bei jeweils i. d. R. sieben Wärmestromdichten im Bereich 2.000 bis $100.000 \mathrm{~W} / \mathrm{m}^{2}$ durchgeführt.

\section{Ergebnisse}

\section{a) Zustandsbereich der Messungen}

In Tab. 4 sind wichtige Stoffdaten der untersuchten Stoffe enthalten. Die kritischen Temperaturen und Drücke wurden für die reinen Stoffe dem VDI-Wärmeatlas entnommen und außerdem mit Realstoffgleichungen $-u$. a. mit NRTL - berechnet. Für die untersuchten Stoffgemische konnten nur die so errrechneten kritischen Daten verwendet werden, da experimentelle Literaturdaten nicht vorlagen und eigene Messungen mit der verwendeten, prinzipiell auch dafür geeigneten Apparatur wegen der zu hohen kritischen Temperaturen der hier untersuchten Stoffe nicht durchfuihrbar waren.

\begin{tabular}{|l|c|c|c|c|}
\hline Bezeichnung & \multicolumn{2}{|c|}{ Aceton } & \multicolumn{2}{c|}{ Ethanol } \\
\hline Summeformel & \multicolumn{2}{|c|}{$\mathrm{C}_{3} \mathrm{H}_{6} \mathrm{O}$} & \multicolumn{2}{c|}{$\mathrm{C}_{2} \mathrm{H}_{6} \mathrm{O}$} \\
\hline Molmasse [g/mol] & \multicolumn{2}{|c|}{58,08} & \multicolumn{2}{c|}{46,07} \\
\hline Kritische Temperatur [K] & 234,95 & 235,05 & 243,05 & 240,77 \\
& {$[4]$} & (NRTL) & {$[4]$} & (NRTL) \\
\hline Kritischer Druck [bar] & 47 & 47,01 & 63,8 & 61,48 \\
& {$[4]$} & (NRTL) & {$[4]$} & (NRTL) \\
\hline Reinheit laut Hersteller & \multicolumn{2}{|c|}{99,9} & \multicolumn{2}{|c|}{99,9} \\
\hline
\end{tabular}

Tab. 1: Daten der verwendeten Versuchsstoffe

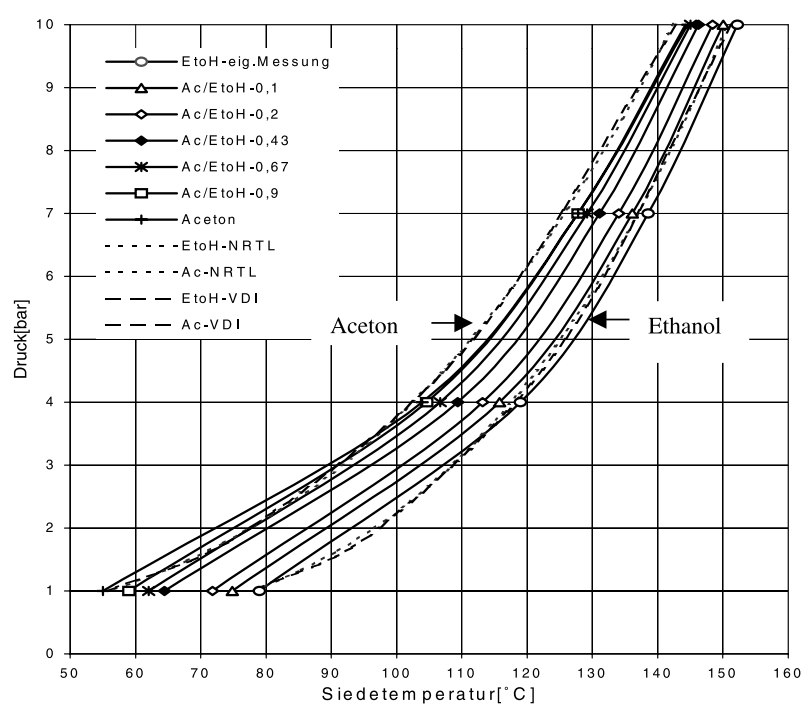

Abb. 2: Zustandsbereich der Messungen, gemessene Dampfdruckkurven der reinen Stoffe und untersuchten Gemische im Vergleich zu den Reinstoffdaten aus VDI-Wärmeatlas [4] und berechneten Daten mit der Methode NRTL.

In Abb. 2 sind die Siedezustände der untersuchten reinen Stoffen und Gemische, bei denen Wärmeübergangsmessungen durchgefuihrt wurden, dargestellt. Jeder der mit hohlen Symbolen eingezeichneten Punkten entspricht einer Messreihe verschiedener Wärmestromdichten bei konstantem Druck. Die fein gestrichelten Kurven stellen die mit der Methode NRTL gerechneten Dampfdruckkurven der reinen Versuchsstoffe dar. Zum Vergleich sind grob gestrichelt auch die Dampfdruck- 
kurven nach VDI-Wärmeatlas für die reinen Komponenten eingezeichnet. Die Übereinstimmung zwischen den mit NRTL gerechneten Dampfdruckkurven und den Literaturwerten nach [4] für Aceton und Ethanol ist relativ gut. Die Abweichungen betragen bei 1 bar 1,5 $\mathrm{K}$ für Ethanol und 1,02 K fuir Aceton und bei 10 bar 0,65 K fuir Ethanol und 1,6 K fuir Aceton. Die Unterschiede zwischen den eigenen Messpunkten und den mit NRTL gerechneten Daten sind von derselben Größenordnung. Sie betragen bei 1 bar $1 \mathrm{~K}$ für Ethanol und 0,5 K für Aceton und bei 10 bar 1,5 $\mathrm{K}$ für Ethanol und 1,6 K für Aceton.

\section{b) Einfluss der Wärmestromdichte auf den Wärme- übergangskoeffizienten (WÜK)}

In Abb. 3 sind die Messergebnisse für die reinen Versuchsstoffe Aceton und Ethanol bei den untersuchten Drücken und Wärmestromdichten in doppellogarithmischer Darstellung aufgetragen. Die darin mit vollen Symbolen zum Vergleich dargestellten Literaturwerte [4] sind auf die in dieser Arbeit untersuchten Drücke und Wärmestromdichten umgerechnet. Außerdem sind darin Messungen von Bonilla und Perry bei 1 bar [6] und von Alpay und Hakan bei 4 bar [7] für Aceton enthalten. Tabelle 2 enthält die Abweichungen der Vergleichswerte aus der Literatur von den eigenen Messungen bei ausgewählten Wärmestromdichten. Wie man sieht, ergibt sich dabei die geringste Abweichung bei großen Wärmestromdichten sowohl für Aceton als auch für Ethanol. Ausnahme davon ist der von Alpay und Hakan angegebene Aceton-Wert bei

. Für Aceton kann aber auch im VDI-Wärmeatlas [4] selbst für den Referenzzustand $\left(\dot{q}=20 \mathrm{~kW} / \mathrm{m}^{2}, p^{*}=0,1\right.$ und $\left.R_{a 0}=0,4 \mu \mathrm{m}\right)$ kein genauer Wert angegeben werden. Die dort angegebene Schwankungsbreite bewegt sich zwischen $=3.200$ und $4.700 \mathrm{~W} / \mathrm{m}^{2}$. Es ist nicht auszuschließen, dass Spuren chemische Zersetzungen des Acetons an den in der Regel verwendeten, dabei möglicherweise katalytisch wirkenden Heizrohren aus Kupfer stattfanden und die relativ hohe Streubreite der Messwerte verursachten. In dieser Arbeit hat sich gezeigt, dass der obere Wert $\alpha=$ $4.700 \mathrm{~W} / \mathrm{m}^{2}$ aus [4] besser zu den eigenen Messergebnissen passt.

\begin{tabular}{|c|l|r|r|r|r|r|r|}
\hline \multirow{3}{*}{$\begin{array}{c}\text { Druck } \\
\text { [bar] }\end{array}$} & \multirow{2}{*}{ Stoff } & \multicolumn{5}{|c|}{ Vergleichsquellen } \\
\cline { 2 - 8 } & & VDI-Wärmeatlas [4] & \multicolumn{2}{|c|}{ Alpay und Hakan [7] } & \multicolumn{2}{|c|}{ Bonilla und Perry [6] } \\
\cline { 2 - 8 } & & $\dot{q}=4 \mathrm{~kW}$ & $\dot{q}=100 \mathrm{~kW}$ & $\dot{q}=4 \mathrm{~kW}$ & $\dot{q}=100 \mathrm{~kW}$ & $\dot{q}=4 \mathrm{~kW}$ & $\dot{q}=100 \mathrm{~kW}$ \\
\hline \multirow{2}{*}{4} & Aceton & $104 \%$ & $4,5 \%$ & -- & -- & $22,2 \%$ & $19,3 \%$ \\
& Ethanol & $1,1 \%$ & $5,3 \%$ & -- & -- & -- & -- \\
\hline \multirow{2}{*}{4} & Aceton & $32,5 \%$ & $13,7 \%$ & $46 \%$ & $268 \%$ & -- & -- \\
& Ethanol & $25 \%$ & $25 \%$ & -- & -- & -- & -- \\
\hline 7 & Aceton & $37,6 \%$ & $6,5 \%$ & -- & -- & -- & -- \\
& Ethanol & $40 \%$ & $30 \%$ & -- & -- & -- & -- \\
\hline \multirow{2}{*}{10} & Aceton & $22,8 \%$ & $5,5 \%$ & -- & -- & -- & -- \\
& Ethanol & $41 \%$ & $17 \%$ & -- & -- & -- & -- \\
\hline
\end{tabular}

Tab. 2: Prozentuale Abweichungen der Daten aus der Literatur und der aus dem VDI-WA [4] umgerechneten Werte von den eigenen Messungen.

In Abb. 4 und 5 sind die Ergebnisse der systematischen Wärmeübergangsmessungen an den fünf untersuchten Aceton-Ethanol-Gemischen zusammengefasst. In der doppellogarithmischen Auftragung des Wärmeüber-
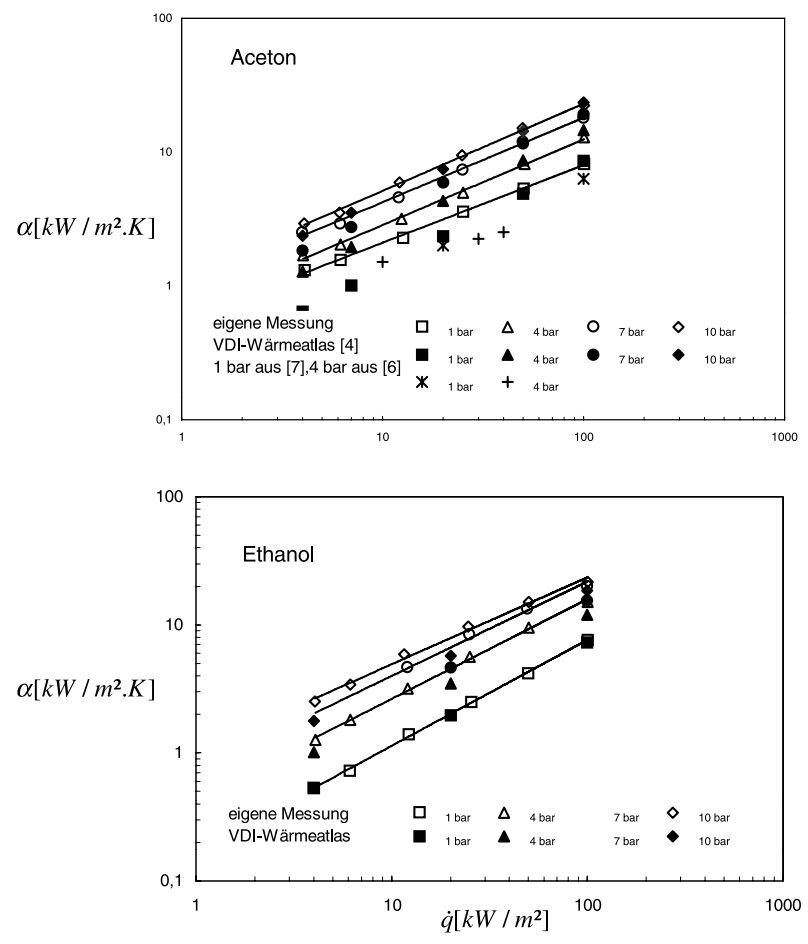

Abb. 3: Wärmeübergangskoeffizient (WÜK) in Abhängigkeit von der Wärmestromdichte (WSD) für die reinen Komponenten Aceton und Ethanol bei verschiedenen Drücken. Vergleich der Ergebnisse dieser Arbeit (hohle Symbole) mit Messwerten von Bonilla und Perry [6] und Alpay und Hakan [7] für Aceton und mit Werten aus dem VDI-Wärmeatlas [4] für beide reinen Stoffe.

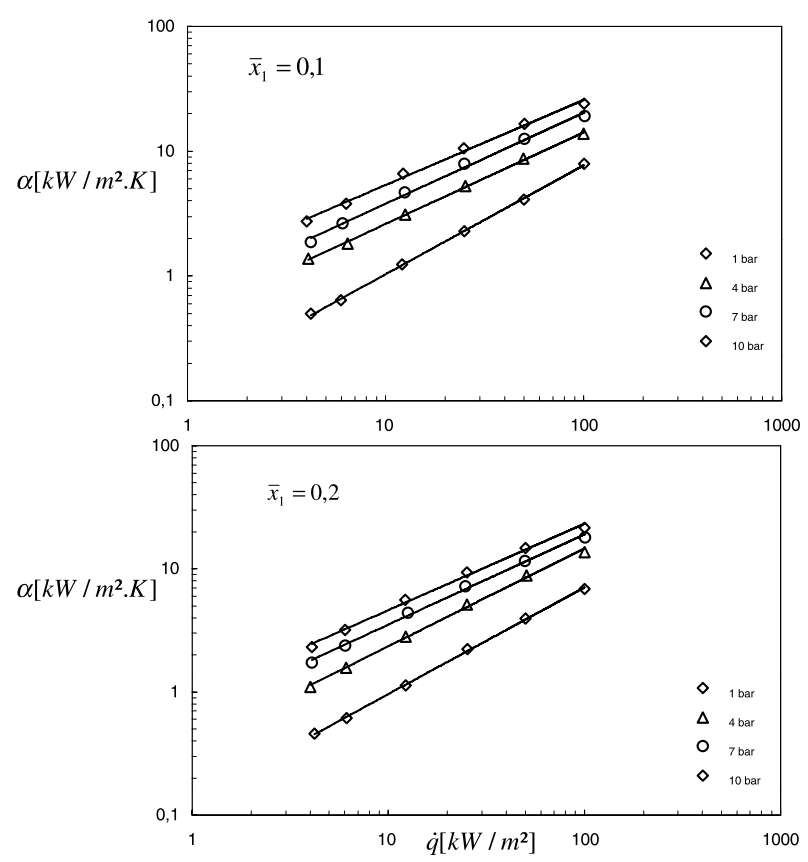

Abb. 4: WÜK $\alpha$ in Abhängigkeit von der WSD für die Aceton-EthanolGemische mit $\bar{x}_{1}=0,1$ (oben) und 0,2 (unten) bei den untersuchten Drücken.

gangskoeffizienten in Abhängigkeit von der Wärmestromdichte mit dem Siededruck als Parameter ergeben sich auch für die Gemische bei allen untersuchten Drücken erwartungsgemäß in guter Näherung Ausgleichsgeraden allerdings mit Steigungen, die nur wenig geringer als die der reinen Stoffe sind. Die Übereinstimmung der in Abb. 5 eingezeichneten Messwerte von Bonilla und Perry [6] mit den eigenen Messwerten für die Stoffmischung ist sowohl bezüglich der $\alpha$-Werte 
wie auch bezüglich der Steigung relativ gut, wenn man diese Abweichungen mit den in Tab. 2 dargestellten Unterschieden für die reinen Stoffe vergleicht. Die Abweichung der Literaturwerte von den eigenen Messwerten beträgt z. B. 19,3\% bei und $22,2 \%$ bei $\dot{q}=20 \mathrm{~kW} / \mathrm{m}^{2}$. Die Steigung der $\alpha, q-\mathrm{Ge}$ raden von Bonilla und Perry stimmt im Rahmen der Ablesegenauigkeit für diese Aceton/Ethanol-Mischung mit der der eigenen Messungen bei 1 bar sogar gut uiberein.

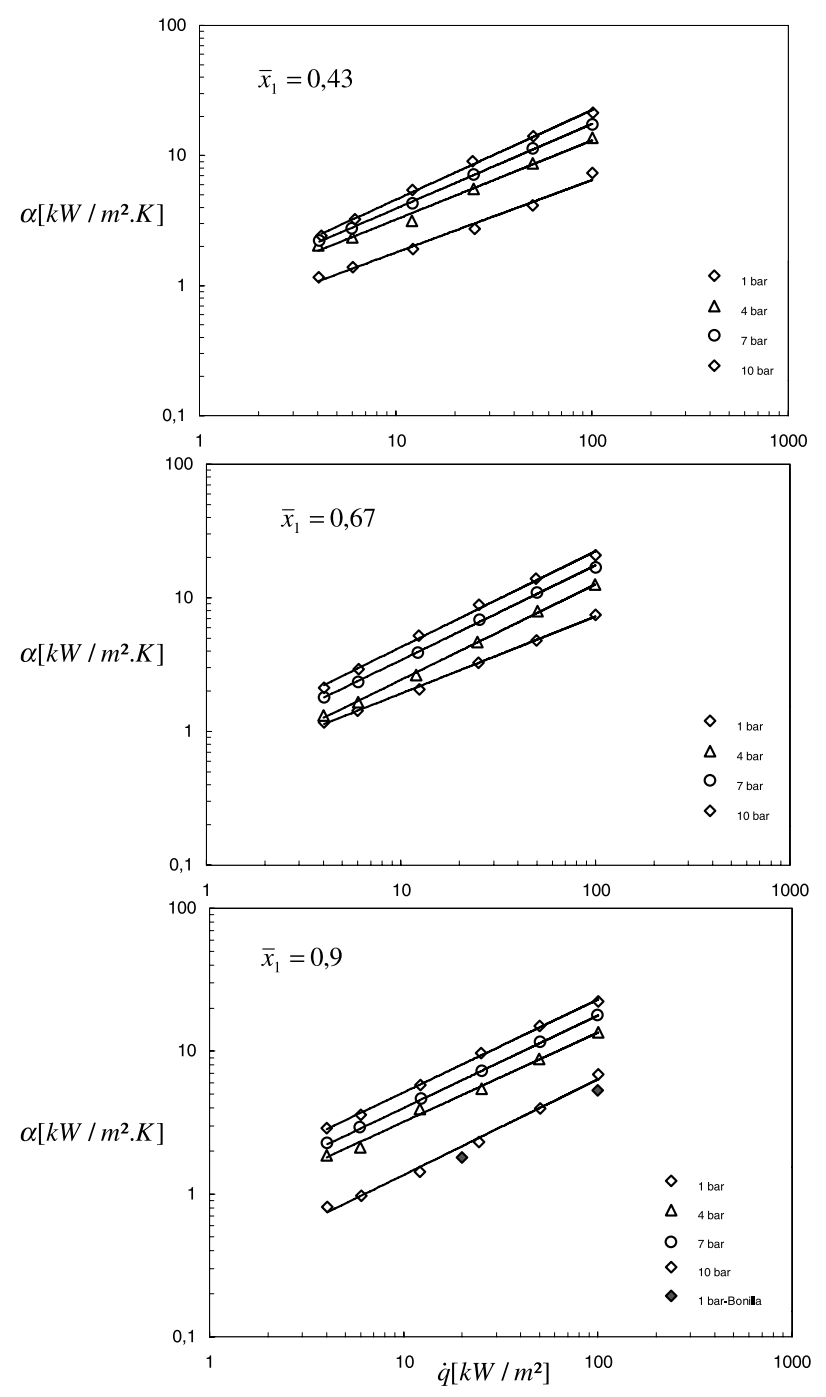

Abb. 5: WÜK in Abhängigkeit von der WSD für die Aceton-EthanolGemische $\bar{x}_{1}=0,43 ; 0,67$ und 0,9 bei den untersuchten Drücken. Vergleich zwischen eigener Messung und Messwerten von Bonilla und Perry [6] bei 1 bar und $\bar{x}_{1}=0,9$.

In den Abb. 6 und 7 wird die Steigung n der $\alpha, \dot{q}$-Geraden näher betrachtet. In Abb. 6 ist in Abhängigkeit vom normierten Druck für die reinen Stoffe vergleichsweise zu den gerechneten Werten nach der bekannten Gleichung

und analogen Werten aus der Literatur [6,7] dargestellt. Man sieht, dass die Steigungen der eigenen $\alpha, \dot{q}$-Geraden durchgehend flacher sind als die nach Gl. (7). Vergleichsweise sind die aus den zur Verfuigung stehenden Literaturdaten ermittelten Steigungen in Abb. 6 mit eingezeichnet. Es ist zu erkennen, dass die aus den Messungen von Alpay und Hakan bei 4 bar entnomme- ne Steigung [7] mit den eigenen Messungen sehr gut übereinstimmt, die Steigungen aus den Messungen von Bonilla und Perry bei 1 bar [6] liegen jedoch noch oberhalb der mit Gl. (7) errechneten Steigungen.

Erwartungsgemäß sollten die Gemischisobaren deutlich flacher als die Reinstoffisobaren sein, besonders bei hohen Drücken über $50 \%$ des kritischen Druckes und bei starkem Gemischeffekt. In Abb. 7 ist nun in Abhängigkeit von der Konzentration für alle untersuchten Stoffgemische bei den vier untersuchten Absolutdrücken dargestellt. Die Reinstoffdaten aus Abb. 6 sind mit eingezeichnet. Es wird deutlich, dass zwischen den Steigungen für reine Stoffe und jenen für Gemische nicht, wie erläutert, der sonst - bei höheren Drüicken und starkem Gemischeffekt - deutliche Unterschied auftritt. Da die Messungen bei geringen normierten Drüicken von 1,5\% bis $15,6 \%$ des kritischen Druckes durchgefuihrt worden sind, ist diese Übereinstimmung nicht unplausibel.

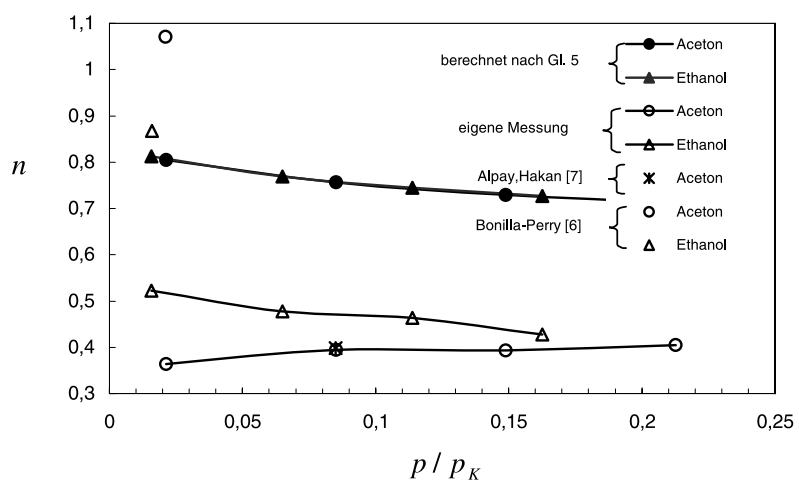

Abb. 6: Exponent n der Wärmestromdichte in Abhängigkeit vom normierten Siededruck für die untersuchten reinen Stoffe.

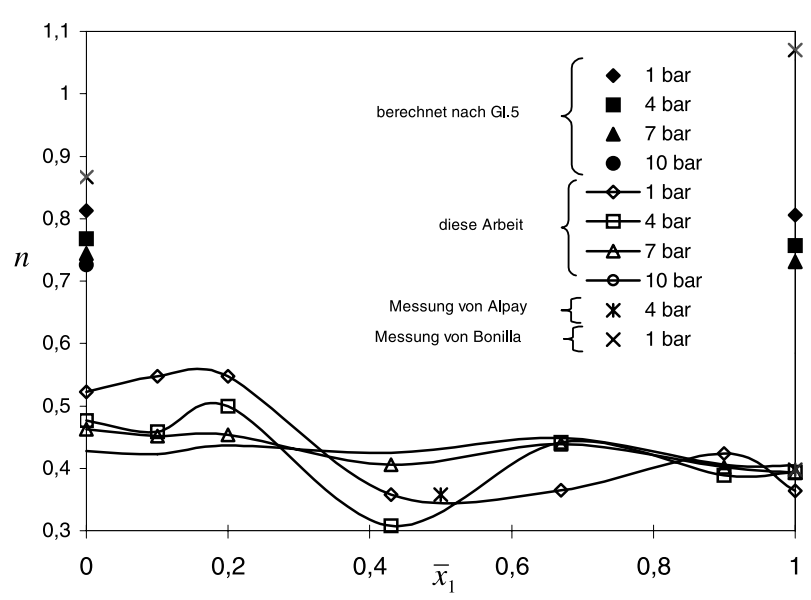

Abb. 7: Exponent der Wärmestromdichte $n$ bei den untersuchten Drücken in Abhängigkeit vom Aceton-Molenbruch für das untersuchte Stoffpaar.

\section{c) Einfluss der Konzentration auf den wüK}

In Abb. 8 sind die Messwerte für die Wärmestromdichten 4.000, 20.000 und $100.000 \mathrm{~W} / \mathrm{m}^{2}$ mit dem Druck 1 bis 10 bar als Parameter in Abhängigkeit vom Molenbruch des leichter Siedenden in der siedenden Flüssigkeit dargestellt. Man erkennt im gesamten untersuchten Druckbereich der Messungen einen relativ schwachen Gemischeffekt und eine relativ große Streuung der Messwerte. Mit fallenden Wärmestromdichten und 
Siededrücken wird der Gemischeffekt dabei schwächer und die Streuung größer. Bei Wärmestromdichte von $100.000 \mathrm{~W} / \mathrm{m}^{2}$ ist ein schwacher Gemischeffekt bei allen Drücken zu beobachten, bei den niedrigen Wärmestromdichten 20.000 und $4.000 \mathrm{~W} / \mathrm{m}^{2}$ nur noch bei den jeweils höchsten untersuchten Siededrücken. Auch die mit eingezeichneten Messpunkte von Bonilla und Perry bei 1 bar und verschiedenen Konzentrationen in Abb. 8a bestätigen den sehr schwachen Gemischeffekt. Eine quantitative Betrachtung des Gemischeffektes bei allen Drücken ist deshalb unter Berüicksichtigung auch der Messgenauigkeit nur bei hohen Wärmestromdichten sinnvoll. Im Folgenden werden die Daten bei der höchsten gemessenen Wärmestromdichte $\dot{q}=100 \mathrm{~kW} / \mathrm{m}^{2}$ näher betrachtet. Um den Gemischeffekt möglichst genau darzustellen, wurden verschiedene Darstellungsvarianten gewählt. Die Abb. 9 bis 11 enthalten verschiedene Darstellungen des Absolutwertes $\alpha$ sowie der normierten wÜKen und $\alpha / \hat{\alpha}$ nach Gleichungen (6) als Funktion des Molenbruchs der siedenden Flüssigkeit $\bar{x}_{1}$ mit dem absoluten Druck $p$ und dem normierten Druck als Parameter.

a)
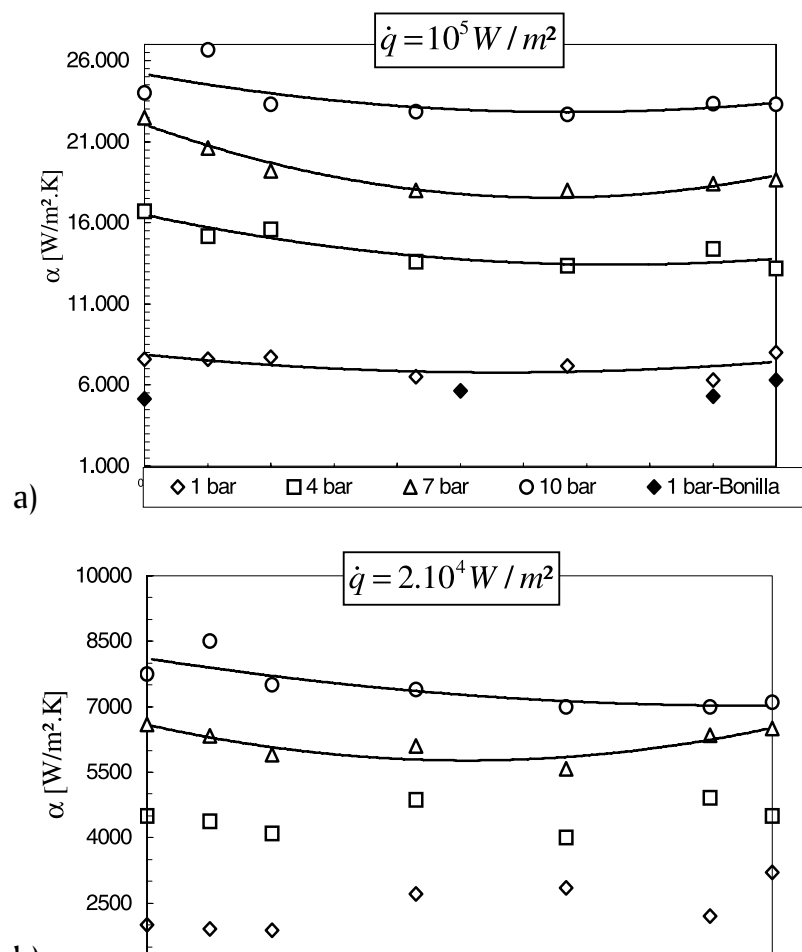

b)

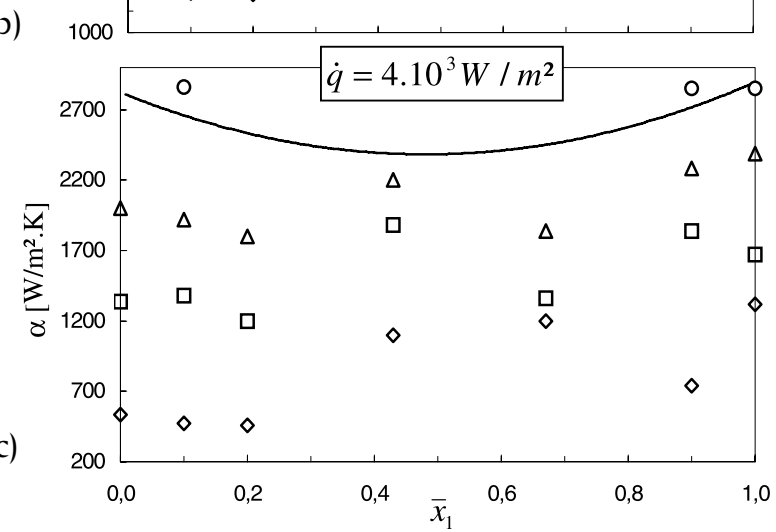

Abb. 8: Wärmeübergangskoeffizient in Abhängigkeit von Aceton-Molenbruch der siedenden Flüssigkeit bei verschiedenen Wärmestromdichten und Drücken. Vergleich zwischen eigener Messung und Bonilla und Perry [6] bei 1 bar und $100 \mathrm{~kW} / \mathrm{m}^{2}$.
Die Abb. 9 bis 11 zeigen die Abhängigkeit des reduzierten WÜKen $\alpha / \alpha_{i d}$ bzw. $\alpha / \hat{\alpha}$, auch ,relative Verschlechterung" genannt, von der Konzentration $\bar{x}_{1}$ bei konstanter WSD $\dot{q}=100 \mathrm{~kW} / \mathrm{m}^{2}$ mit $p=1,4,7$ und 10 bar bzw. $\quad=0.05,0.10,0.15$ und 0.20 als Parameter. In Abb. 9 ist $\alpha / \alpha_{i d}$ nach Gl. (6b) ausgehend von interpolierten Werten aus den $\alpha-\dot{q}$-Geraden (Abb. 3 bis 5) dargestellt. Es ist zu erkennen, dass die relative Verschlechterung maximal zwischen ca. 86 und $90 \%$ von $\alpha_{i d}$ liegt. Erwartungsgemäß sollte diese maximale Verschlechterung im Bereich des Minimums von mit steigendem Druck zunehmen [3]. Hier ist sogar ein umgekehrter Effekt zu beobachten: Bei $p=1$ bar ist die Verschlechterung stärker als bei $p=10$ bar. Man sieht auch, dass sich am rechten Konzentrationsrand ein azeotroper Punkt andeutet, der sich mit steigendem Druck immer deutlicher ausprägt und der durch Messungen und Berechnungen der Siedelinse [9] bestätigt wird. Auch bei den Messungen von Alpay und Hakan ist der azeotrope Punkt erst bei $\quad=5$ bar andeutungsweise zu sehen.

Die Darstellung in Abb. 10 ist analog zu der in Abb. 9, mit der zusätzlichen Darstellung des normierten WÜKen nach Gl. (6a). Um die Streuung der Messwerte besser zu quantifizieren, z. B. wegen der Streuung am linken Konzentrationsrand bei $=10$ bar, wurde in Abbildung 10 eine Darstellung gewählt, die auf einer Darstellung des WÜK als Funktion des Druckes von der Form basiert [9]. Das so erhaltene Bild ist erwartungsgemäß qualitativ ähnlich dem in Abb. 9. Allerdings bewegt sich das Maximum der relativen Verschlechterung in einem etwas breiteren Bereich zwischen ca. 0,80 und 0,90 . Eine systematische Druckabhängigkeit ist auch hier nicht erkennbar. Wie in Abb. 9 ist die relative Verschlechterung auch hier bei $p=1$ bar höher als bei =10 bar, was bei höheren Drücken bekanntlich umgekehrt ist. Dort nimmt die relative Verschlechterung mit steigendem Druck in der Regel zu. Das zu Abb. 9 bezüglich des angedeuteten azeotropen Punktes Gesagte gilt hier analog. Die gestrichelten Linien in Abb. 10 stellen die relative Verschlechterung des Wärmeübergangs in der Körnerschen Darstellung $\alpha / \hat{\alpha}$ nach Gl. (6a) dar. Man sieht, dass sich in dieser Darstellung geringere relative Verschlechterungen ergeben als mit der Happelschen Darstellung nach Gl. (5), die auch in den Abb. 9 und 11 verwendet wird. Der Unterschied zwischen den beiden Darstellungen beträgt bis zu ca. 1,5\% bei 1 bar und bis zu ca. $1 \%$ bei 10 bar, jeweils bezogen auf $\alpha_{i d}$.

In Abb. 11 ist uiber $\bar{x}_{1}$ analog zu Abb. 9 und 10 aber abweichend davon mit dem normierten Druck $p^{*}$ als Parameter dargestellt. Für diesen Zweck sind zunächst die Drücke für alle untersuchten Gemische mit den Daten aus Abb. 9 auf die jeweiligen normierten Druicke umgerechnet und sodann die $\alpha$-Werte bei konstantem aus den entsprechenden Ausgleichskurven entnommen worden. Die relative Verschlechterung $\alpha / \alpha_{i d}$ bewegt sich hier im Minimum zwischen ca. 86 und $88 \%$ von $\alpha_{i d}$. In dieser Darstellung ergibt sich die - wenn auch sehr schwache so doch erwartungsgemäße - Zunahme der relativen Verschlechterung mit steigendem normierten Druck, anders als in den Abbildungen 9 und 10 mit 
dem Absolutdruck als Parameter. Auch hier deutet sich der azeotrope Punkt am rechten Konzentrationsrand an, deutlich beim höchsten nicht erkennbar beim niedrigsten dargestellten normierten Druck. Die Messwerte beim höchsten normierten Druck $\quad=0,2$ sind vollstän-

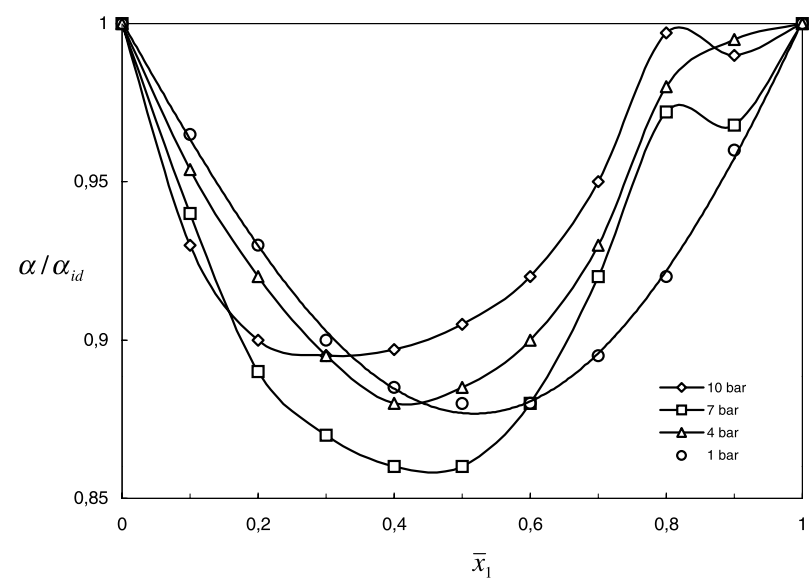

Abb. 9: Reduzierter Wärmeübergangskoeffizient

für das untersuchte Stoffpaar Aceton-Ethanol in Abhängigkeit vom Molenbruch $\bar{x}_{1}$ bei konstanter Wärmestromdichte und verschiedene Drücken. Die Symbole stellen keine direkten Messwerte sondern mit Gl. (6) aus Abb. 3 bis 5 umgerechnete Werte dar.

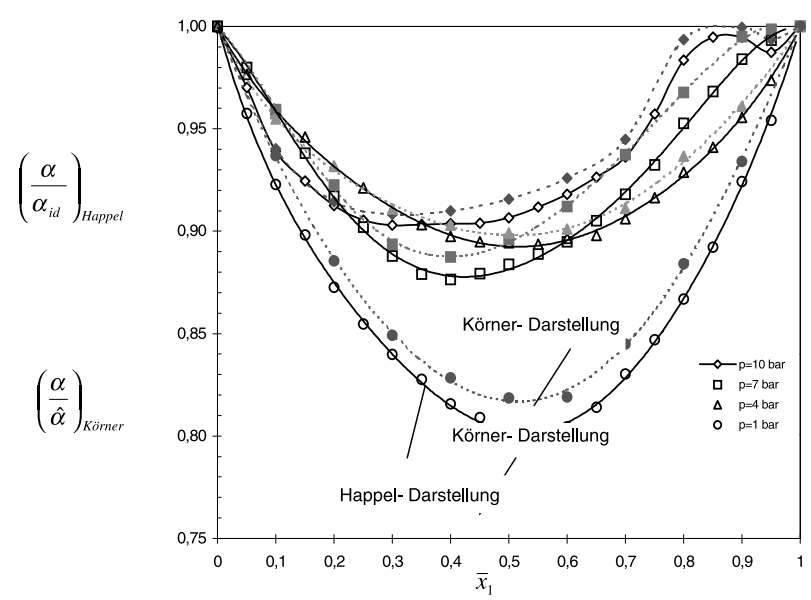

Abb. 10: Reduzierte WÜKen $\alpha / \alpha_{i d}$ und $\alpha / \hat{\alpha}$ für das untersuchte Stoffpaar Aceton-Ethanol in Abhängigkeit vom Molenbruch $\bar{x}_{1}$ bei konstanter Wärmestromdichte und verschiedenen Drücken. Die Symbole stellen keine direkten Messwerte sondern mit Gl. (6) aus Diagrammen $\alpha=f(p)$ [9] umgerechnete Werte dar.

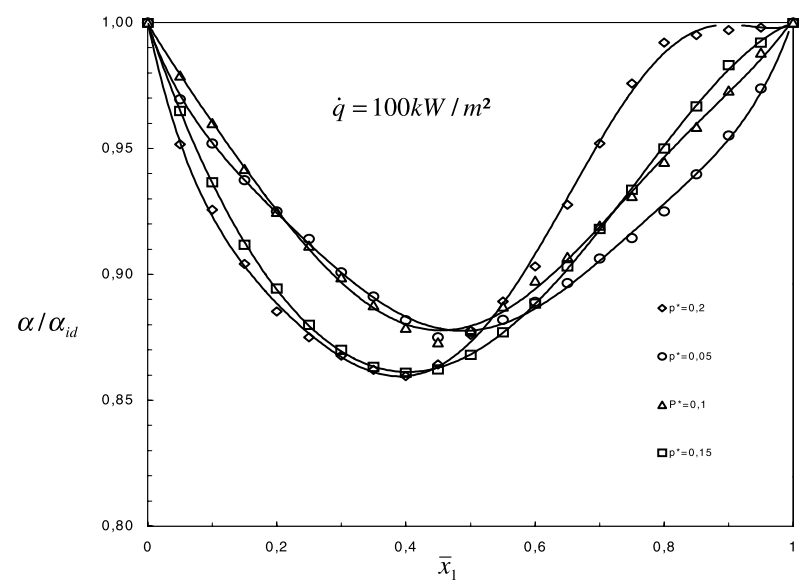

Abb. 11: Wärmeübergangskoeffizient und der reduzierte WÜK

für das untersuchte Stoffpaar Aceton-Ethanol in Abhängigkeit vom Molenbruch $\bar{x}_{1}$ bei konstanter Wärmestromdichte und verschiedenen normierten Drücken. Die Symbole stellen keine direkten Messwerte sondern mit Gl. (6) aus Diagrammen $\alpha=f\left(p^{*}\right)$ [9] umgerechnete Werte dar. dig extrapoliert, da der höchste untersuchte Druck $p=10$ bar fuir alle untersuchten Gemische durchgehend leicht darunter liegende normierte Drücke ergibt.

Das Vorhandensein eines mit steigendem Druck zunehmend deutlich ausgeprägten azeotropen Punktes am rechten Konzentrationsrand bestätigt sich in den Messungen und Berechnungen des Phasengleichgewichtes Dampf/fluissig. Die Ergebnisse der Phasengleichgewichtsmessungen und der entsprechenden NRTL-Berechnungen sind in Abb. 12 für die vier untersuchten Drücke dargestellt. Für die Gemische wurden die Konzentrationen der Flïssigkeit und des Dampfes jeweils bei jedem Druck experimentell ermittelt [9]. Während die Flüssigkeitskonzentrationen sehr zuverlässig ermittelt werden konnten, sind die gemessenen Dampfkonzentrationen unsicher, wegen Kondensationsproblemen in der Probenahmeleitung. Deshalb wurden die Dampfkonzentrationen zusätzlich bei allen vier untersuchten Drücken mit dem Prozesssimulationsprogramm CHEMCAD berechnet. Es ist bekannt, dass der Flüssigkeitszustand beim Gemischsieden umso mehr überhitzt ist im Vergleich zum thermodynamischen Gleichgewicht, je niedriger der Druck ist. Deshalb liegen die gemessenen Konzentrationen bei allen Drücken erwartungsgemäß oberhalb der berechneten Siedelinsen, besonders bei $\mathrm{p}=1$ bar. Auch ist erkennbar, dass sich ab 4 bar am rechten Konzentrationsrand ein azeotroper Punkt andeutet, der bei 10 bar schon deutlich ausgeprägt ist.

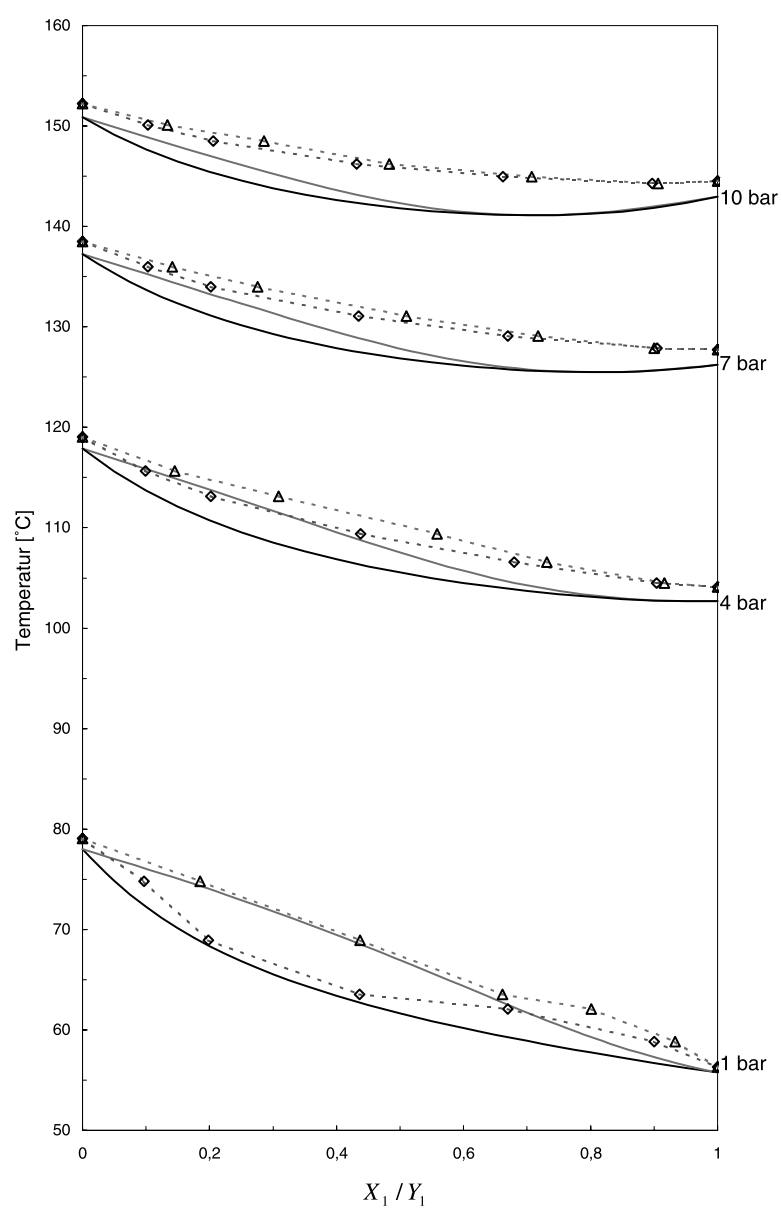

Abb. 12: Siedelinse des untersuchten Stoffsystems Aceton-Ethanol berechnet mit NRTL bei den vier untersuchten Drücken. Die eingezeichneten Symbole mit gestrichelten Linien stellen die eigenen gaschromatographisch ermittelten Dampf- und Flüssigkeitskonzentrationen dar. 


\section{Zusammenfassende Diskussion der relativen Verschlechterung des Wärme- übergangskoeffizienten}

Erwartungsgemäß wurde bei konstantem Druck das erwartete Minimum des Wärmeübergangskoeffizienten $\alpha$ im mittleren Konzentrationsbereich der siedenden Flüssigkeit etwa bei $\quad=0,3$ bis 0,5 gefunden. Die relative Verschlechterung des Wärmeübergangs $\alpha / \alpha_{i d}$ bewegt sich in diesem Konzentrationsbereich zwischen max. 0,80 bis 0,88 bei 1 bar und max. 0,89 bis 0,91 bei 10 bar. Anders als erwartet aber verhält sich die Druckabhängigkeit von $\alpha / \alpha_{i d}$ : Während $\alpha / \alpha_{i d}$ bei hohen Drücken grundsätzlich mit steigendem Druck zunimmt, ergab sich aus den Messergebnissen dieser Arbeit eine Abnahme von 1 bar bis 10 bar. Durch Auswertung nach zwei verschiedenen Methoden, zum einen Interpolation der Messwerte aus $\alpha, \dot{q}$-Daten und zum anderen aus Daten, wurde versucht zu klären, ob dieser unerwartete Befund möglicherweise im Rahmen der Messgenauigkeit liegt. Ein Vergleich der Ergebnisse der beiden Auswertungsmethoden in Kapitel 4.5 zeigte, dass zwar beträchtliche Streuungen der Messdaten insbesondere bei 1 bar zu berücksichtigen sind, die Umkehr der Druckabhängigkeit damit aber nicht erklärt werden kann. Anschließende Korrelationsberechnungen mit der Gleichung von Stephan und Körner in Kapitel 5.2 ergeben einen analogen Druckeinfluss und bestätigen somit indirekt den theoretisch zunächst nicht erklärbaren Sachverhalt.

Übrigens tritt dieser Befund in der normierten Darstellung bei = konstant nicht auf. Da die normierte Darstellung dem thermodynamischen Korrespondenzprinzip entspricht, hat sie deshalb eine andere Aussagekraft als die Darstellung mit dem Absolutdruck als Parameter. Beide Befunde können demnach gleichzeitig richtig sein: Die Darstellung $\alpha / \alpha_{i d}=f\left(\bar{x}_{1}\right)$ mit als Parameter bestätigt den aus der Literatur bekannten Sachverhalt, dass die relative Verschlechterung mit steigendem Druck zunimmt. Erwartungsgemäß ist die Zunahme bei diesem Stoffsystem nur sehr schwach. Dass dieser Sachverhalt bei Darstellung mit dem Absolutdruck als Parameter scheinbar widerlegt wird, kann zum einen auf den beträchtlichen Unterschied in den kritischen Punkten der reinen Komponenten - zumindest als eine Ursache - zurückgefuihrt werden. Der Unterschied in den kritischen Drüicken der reinen Komponenten beträgt immerhin ca. 15 bar. Dadurch wird die Realität in der Darstellung bei konstantem Absolutdruck p verzerrt. Die wesentliche Erklärung aber liefern die in Abb. 12 dargestellten Konzentrationsmessungen und -berechnungen: Dabei zeigte sich, dass dieses Stoffsystem einen azeotropen Punkt aufweist, der aber bei niedrigen Drüicken unter 4 bar nicht zu beobachten ist. Dieser azeotrope Einfluss uiberlagert den Gemischeffekt und bewirkt eine Verbesserung des Wärmeübergangs, die sich der Verschlechterung im rechten Konzentrationsbereich bei hohen Acetongehalten der Verschlechterung überlagert und deren Zunahme mit steigendem Druck in eine Abnahme verkehrt (Abb. 9 und 10) bzw. fast kompensiert (Abb. 11).

\section{Literatur}

[1] K. STEPHAN und M. KÖRNER: Berechnung des Wärmeübergangs verdampfender binärer Flüssigkeitsgemische. Chem. Ing. Technik 41 (7), S. 409-416, 1969

[2] O. HAPPEL: Wärmeübergang bei der Verdampfung binärer Gemische im Gebiet des Blasen- und Übergangssiedens. Dissertation, Ruhr- Universität Bochum, 1975

[3] J. SCHMADL: Wärmeübergang beim Blasensieden binärer Stoffgemischen unter hohem Druck. Dissertation, Universität Fridericiana Karlsruhe, 1982

[4] VDI-WÄRMEATLAS. VDI-Verlag Düsseldorf, 7. Auflage, Abschnitt Hab, 1994

[5] M. KÖRNER: Beitrag zum Wärmeübergang bei der Blasenverdampfung binärer Gemische. Dissertation, RheinischWestfälischen Technischen Hochschule Aachen, 1967

[6] C. F. BONILLA und C. W. PERRY: Heat Transmission to Boiling Binary Liquid Mixtures. Trans. Am. Inst. Chem. Engrs. 37, S. 685-705, 1941

[7] H. ALPAY and F. BALKAN: Nucleate pool boiling performance of acetone-ethanol and methylenechloride-ethanol binary mixtures. Department of Chemical Engineering, Ege University, Izmir, Turkey, 1988-1989

[8] J. SCHMADL und P. IGNASZEWSKI: Inbetriebnahme einer Präzisions-Apparatur für Wärmeübergangsmessungen beim Sieden reiner Stoffe und Nachrüstung für Stoffgemische. Wissenschaftliche Beiträge der Techn. Fachhochschule Wildau 02/1998

[9] R. WITTL: Wärmeübergang beim Blasensieden des binären Stoffsystems Aceton-Ethanol, Diplomarbeit TFH Wildau, 2001.

[10]J. SCHMADL und R WITTL: Korelation des Wärmeübergangskoeffizienten mit Gleichungen vom Typ Stephan-Körner beim Sieden von Stoffgemischen aus Aceton und Ethanol, Wissenschaftliche Beiträge TH Wildau, Heft 2002.

\section{Symbole und Formelzeichen}

\begin{tabular}{|c|c|c|}
\hline$C_{1}, C_{2}$ & Konstanten & - \\
\hline$n$ & Exponent der Wärmestromdichte & \\
\hline $\mathrm{p}_{\mathrm{k}}$ & kritischer Druck & bar \\
\hline & normierter Druck & bar \\
\hline , WSD & Wärmestromdichte & $\mathrm{kW} / \mathrm{m}^{2}$ \\
\hline$T$ & Temperatur & K \\
\hline$\alpha$, WÜK & $\begin{array}{l}\text { Wärmeübergangskoeffizient } \\
\text { mittlere Wärmeuibergangs- } \\
\text { koeffizienten der reinen Stoffe } \\
\text { nach Gl. (6) }\end{array}$ & $\mathrm{W} / \mathrm{m}^{2} \cdot \mathrm{K}$ \\
\hline $\bar{x}_{1}$ & $\begin{array}{l}\text { Molenbruch des leichter Sieden- } \\
\text { den in der siedenden Flüssigkeit }\end{array}$ & - \\
\hline $\bar{y}_{1}$ & $\begin{array}{l}\text { Molenbruch des leichter Sieden- } \\
\text { den im Dampf }\end{array}$ & \\
\hline VDI-WA & VDI-Wärmeatlas & - \\
\hline
\end{tabular}

\section{Autoren}

\section{Prof. Dr.-Ing. Josef Schmadl \\ Dipl.-Ing. (grad.) Romel Wittl}

Technische Fachhochschule Wildau

Technikum für Thermische Verfahrenstechnik

Tel. (0 33 75) 508-110, Fax (0 33 75) 508-906

E-Mail: jschmadl@igw.tfh-wildau.de 